\title{
Contributors to the 2018 IMIA Yearbook of Medical Informatics
}

\author{
Editors: \\ John H. Holmes \\ University of Pennsylvania \\ Perelman School of Medicine \\ Philadelphia, Pennsylvania, USA \\ jhholmes@pennmedicine.upenn.edu

Brigitte Séroussi
Sorbonne University \& LIMICS UMR S 1142
Tenon Hospital, AP-HP
Paris, France
brigitte.Seroussi@aphp.fr
Lina F.SOualmia
Normandie University, UNIROUEN, LTIIS
Rouen, France
Lina.Soualmia@chu-roven.fr

Editorial Assistant:

Martina Hutter

Heidelberg University Hospital - ZIM

Heidelberg, Germany

yearbook@imia-services.org

\section{Best Papers Editor:}

Adrien Ugon

ESIEE-Paris

adrien.ugon@esiee.fr

Advisory Board:

Reinhold Haux

Peter L. Reichertz Institute for Medical Informatics

(PLRI) of TU Braunschweig and Hannover

Medical School

Braunschweig, Germany

reinhold.haux@prri.de

Victor Maojo

Universidad Politécnica de Madrid

Departamento de Inteligencia Arificicial

Facultad de Informatica

Madrid, Spain

vmaoj@@fi.upm.es

George Mihalas

University of Medicine and Pharmacy

Dept. of Medical Informatics

Timisorara, Romania

mihalas@EFMl.info

\author{
Section Editors: \\ Eta S. Berner \\ Department of Health Services Administration \\ University of Alabama at Birmingham \\ Birmingham, AL, USA \\ eberner@uab.edu \\ Meryl Bloomrosen \\ Premier healthcare alliance \\ Washington, DC \\ meryl_bloomrosen@premierinc.com \\ Jacques Bouaud \\ DRCI/AP-HP \& LIMICS UMR S 1142 \\ Paris, France \\ jacques.bouaud@aphp.fr \\ Jean Charlet \\ DRCI/AP-HP \& LIMICS UMR_S 1142 \\ Paris, France \\ jean.charlet@upmc.fr \\ Christel Daniel \\ WIND-DSI/AP-HP \& LIMICS UMR S 1142 \\ Paris, France \\ christel.daniel@aphp.fr \\ Thomas M. Deserno \\ Peter L. Reichertz Insitutute for Medical Informatics \\ (PLRI) of TU Braunschweig and Hannover \\ Medical School \\ Braunschweig, Germany \\ thomas.deserno@plri.de \\ Ferdinand Dhombres \\ Sorbonne University \& LIMICS UMR S 1142 \\ Trousseau Hospital, AP-HP \\ Paris, France \\ ferdinand.dhombres@inserm.fr \\ Kenneth Goodman \\ University of Miami \\ Miami, Florida, USA \\ kgoodman@med.miami.edu \\ Werner Hack \\ UMIT - University for Health Sciences, \\ Medical Informatics and Technology \\ Hall in Tirol, Austria \\ werner.hack@umit.at \\ Alexander Hoerbst \\ UMIT - University of Health Sciences, \\ Medical Informatics and Technology \\ Hall in Tirol, Austrio \\ alexander.hoerbst@umit.at
}

\author{
Rodolphe Thiébaut \\ Bordeaux University \& Inserm UMR 1219 \\ Bordeaux, France \\ rodolphe.thiebaut@u-bordeaux.fr \\ Frantz Thiessard \\ Bordeaux University \& Inserm UMR 1219 \\ Bordeaux, France \\ frantz.thiessard@u-bordeaux.fr \\ Jeremy L. Warner \\ Vanderbilt University \\ Nashville, TN, USA \\ jeremy.warner@vanderbilt.edu \\ Pierre Zweigenbaum \\ LIMSI, CNRS, Université Paris-Saclay \\ Orsay, France \\ pz@limsi.fr
}

Regional Editors:

Dr. Kyung-Hee Cho

APAMI

President@APAMI.org

Ghislain B. Kovematchoua Tchuitcheu

HELINA

kovematchoua@helina-online.org

Amado Espinosa

IMIA-LAC President

aespinosa@medisist.com.mx

Najeeb Al-Shorbaji

MENAHIA

shorbajin@gmail.com

Karen Greenwood

AMIA

Bethesda, MD, USA

karen@amia.org

Andre Kushniruk

Digital Health Canada: Canada's Health

Informatics Association

Toronto, ON, Canada

andrek@uvic.ca

Anne Moen

EFMI

anne.moen@medisin.vio.no

IMIA Executive Director:

Elaine Huesing

imia@imia-services.org 